\title{
Miniaturized Sensing Devices for Biomarker Detection
}

Ajeet Kaushik $^{1^{*}}$, Ariel Ruiz ${ }^{1}$, Shekhar Bhansali ${ }^{2}$ and Madhavan Nair ${ }^{1}$

${ }^{1}$ Center of Personalized Nano medicine, Institute of Neuroimmune Pharmacology, Department of Immunology, Herbert Wertheim College of Medicine, Florida International University, Miami, FL 33199, USA.

${ }^{2}$ BioMEMS Microsystems Laboratory, Department of Electrical and Computer Engineering, Florida International Laboratory, Miami, FL 33174, USA.

*Corresponding author: Ajeet Kaushik, Center of Personalized Nanomedicine, Institute of Neuroimmune Pharmacology, Department of Immunology, Herbert Wertheim College of Medicine, Florida International University, Miami, FL 33199, USA, Tel: +1-305-348-1490; E-Mail: akaushik@fiu.edu

Rec Date: Sep 16 2014; Acc Date: Sep 17 2014; Pub Date: Sep 242014

Copyright: (c) 2014 Ajeet Kaushik. This is an open-access article distributed under the terms of the Creative Commons Attribution License, which permits unrestricted use, distribution, and reproduction in any medium, provided the original author and source are credited.

\section{Editorial}

From the advent of glucose sensors, efforts have been made to design and develop new smaller sensors that are able to detect a wide variety of biomarkers. Miniature devices at point-of-care (POC) are becoming a trending subject for research and development in environment and healt monitoring and sensing. The information provided by such devices could revolutionize the fields of health informatics and novel treatment strategies by giving them a new basis. The development of these next generation devices, however, would also require a new class of assays and biosystems which can freely integrate itself into the environment of the analyte in order to retrieve the desired information both accurately and in real-time. The selection of sensing materials, transducer, electronic circuit, sensor design, easy operation, low power consumption, packaging, and automation of sample acquisition is crucial to the development of a desired sensor at POC applications. These challenges can be addressed using nanoenabling components in sensor design and development.

Nanomaterial's have been shown capable of detecting biomarkers at the $\mathrm{pM}$ scale, when arranged in a 1D structure. These nano-sensing platforms are being integrated with Microelectromechanical Systems (MEMS) in order to avoid error and increase precisio through automation. To investigate personalized treatments of particular diseases, the health informatics of each individual is crucial. Because of this, researchers were motivated to investigate and develop wearable POC biosensing systems to monitor personal health in an attempt to improve personalized diagnoses and treatments. For example, an LMP 91000 chip based miniaturized potentiostat has been reconfigured to be capable of performing electrochemical measurements. This potentiostat is further interfaced with a microfluidic manifold, consisting of an electrochemical sensor for the use of detecting biomarkers. The integrated sensing device (Figure 1) has successfully detected biomarkers down to the $\mathrm{pM}$ level and has potential to detect a variety of desired physiologically relevant biomarkers at on-site, infield, and POC [1,2].
The aim of this editorial for journal of biosensors and bioelectronics is to attract researchers to explore the novel sensing platforms including design and development of novel sensing material, detection devices, and related nanostructures.



\section{References}

1. Cruz AF, Norena N, Kaushik A, Bhansali S (2014) A Low-Cost Miniaturized Potentiostat for Point-of-Care Diagnosis. Biosens Bioelectron 62: 249-254.

2. Vasudev A, Kaushik A, Tomizawa Y, Norena N, Bhansali S (2013) An LTCC-based microfluidic system for label-free, electrochemical detection of cortisol. Sensor Actuat B-Chem 182: 139-146. 